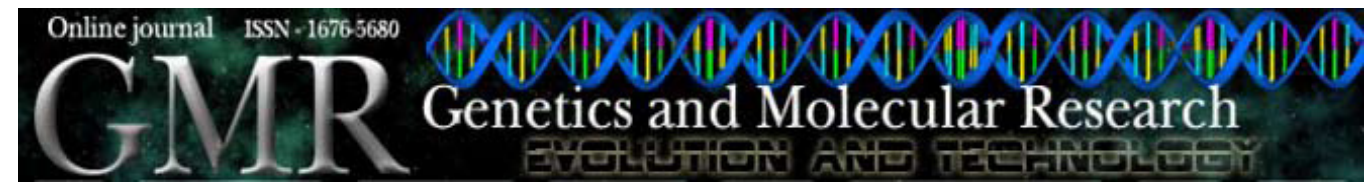

\title{
Highly conserved regions in the 5' region of human olfactory receptor genes
}

\author{
H.F. Tobar ${ }^{2 *}$, P.A. Moreno ${ }^{2,3 *}$ and P.E. Vélez ${ }^{1,2 *}$ \\ ${ }^{1}$ Department of Biology, Universidad del Cauca, Popayán, Colombia \\ ${ }^{2}$ Group of Molecular, Environmental, and Cancer Biology BIMAC, \\ Universidad del Cauca, Popayán, Colombia \\ ${ }^{3}$ School of Systems and Computer Engineering, Universidad del Valle, \\ Santiago de Cali, Colombia \\ *All authors contributed equally to this study. \\ Corresponding author: P.E. Vélez \\ E-mail: pvelez@unicauca.edu.co
}

Genet. Mol. Res. 8 (1): 117-128 (2009)

Received October 27, 2008

Accepted November 28, 2008

Published February 10, 2009

\begin{abstract}
Regulation of human olfactory receptor (hOR) genes is a complex process of control and signalization with various structures and functions that are not clearly understood. To date, nearly 390 functional hOR genes and 462 pseudogenes have been discovered in the human genome. Enhancer models and trans-acting elements for the regulation of different hOR genes are among the few examples of our knowledge concerning regulation of these genes. We looked for upstream control elements that might help explain these complex control mechanisms. To analyze the human olfactory gene family, we looked for functional genes and pseudogenes common to all hOR genes obtained from public databases. Subsequently, we analyzed sequences upstream of the transcription start sites with data mining and bioinformatics tools. We found two highly conserved regions, which we called HCR I and HCR II, upstream of the transcription start sites in $77 \mathrm{hOR}$ genes and 87 pseudogenes. These regions showed possible enhancer functions common to both genes
\end{abstract}


and pseudogenes, an intriguing feature that may be associated with the expression of pseudogenes. Based on these HCRs, we propose a structural model of gene regulation for the olfactory gene family.

Key words: Olfactory receptor gene; Promoter region; Highly conserved region; Regulation; Enhancers

\section{BACKGROUND}

The sense of smell in humans is an important and complex mechanism, where different molecular structures, functions, and regulatory processes converge. The integration of these characteristics allows the detection of many different odorant molecules with high sensitivity and specificity. The responsibility for the perception of smells is assigned to signal transduction proteins in the sensory neuron called olfactory receptors (OR). These proteins make up a larger gene family in many animal species (Buck and Axel, 1991). In the human genome, this gene family is one of the most representatives especially that on chromosome 11 since $40 \%$ of the whole set of human OR (hOR) genes is found in this chromosome (Taylor et al., 2006). Olfactory receptor genes have interesting characteristics that indicate a complex regulation mechanism. Some of them are the presence of a single OR gene in an olfactory neuron (Chess et al., 1994; Malnic et al., 1999), the organization of axons of the olfactory neurons according to the type of receptor that is presented (Wang et al., 1998; Feinstein and Mombaerts, 2004; Barnea et al., 2004), and the presence of functional genes and pseudogenes in different tissues (Zhang et al., 2007).

The regulation of these different structural and functional characteristics needs the integration of specific control mechanisms such as enhancers, silencers, transcription factors, repressors, activator binding sites, and possible unknown mechanisms of control and signalization. The regulation of the OR genes in mice is associated with O/E-like and homeodomain binding sites with possible enhancer function (Michaloski et al., 2006); however, it is not clearly understood. Many studies on olfactory receptor genes in mice show different conserved regions and transcription factors upstream from the transcription start sites (TSS), but it is only for a small number of genes (Qasba and Reed 1998; Sosinsky et al., 2000; Lane et al., 2001; Vassalli et al., 2002; Michaloski et al., 2006; Hoppe et al., 2000, 2003, 2006). All these works taken together show poor understanding of the upstream control elements present in each hOR. Thus, we proposed the examination of the promoter regions of the hOR genes from the HORDE (Human Olfactory Receptor Data Exploratorium) database to establish the existence of cis-acting regulatory elements with a possible role in hOR gene regulation.

\section{MATERIAL AND METHODS}

\section{Database for functional hOR genes}

The hOR genes with a non-coding 5' sequence of $1000 \mathrm{bp}$ corresponding to the pro- 
moter region were downloaded from the HORDE database (Olender et al., 2004), available at the website: http://bioportal.weizmann.ac.il/HORDE/ (HG 18, November 22, 2007). The Tables Browser Tool of the UCSC Genome Browser, http://genome.ucsc.edu (Hinrichs et al., 2006) was used to download the sequences. Subsequently, we defined the extramembrane motifs in the coding region of the gene, according to Zozulya et al. (2001), and defined the functional genes and pseudogenes using the Niimura and Nei (2003) method.

\section{Detection of regularities on the promoter region}

Given the lack of similarity in these upstream regions, multiple alignments were made by using several sequences to search for regularities in the promoter region of the hOR genes. These alignments were conducted via the ClustalW (Thompson et al., 1994) and T-Coffee (Notredame et al., 2000) algorithms.

The conserved regions detected in each alignment were sought in the whole set of hOR genes, and re-aligned again. This step was necessary to find all possible genes with conserved regions.

The possible regulatory function for each conserved region was determined through the BLAST algorithm (Altschul et al., 1997) on the Transcription Regulatory Regions Database (TRRD; Kolchanov et al., 2002).

Finally, the cis-acting regulatory elements with a possible gene regulatory role were examined by means of the weeder prediction tools (Pavesi et al., 2004). The Sequence Logo Tool (Crooks et al., 2004) was used to represent the structural characteristics of each conserved region.

\section{Analysis of chromosomal and phylogenetic clustering}

The definition and classification of chromosomal and phylogenetic clustering were carried out via hOR functional genes and pseudogenes with conserved regions upstream from the transcription start sites. Chromosomal clustering was conducted using information about the gene distribution on the chromosomes or genomic distribution downloaded from the database. For each gene, we defined and implemented a localization nomenclature according to three parameters of distribution in each chromosome: regions, clustering, and sub-clustering, i.e, $\geq 1000 \mathrm{~kb}$ for regions (right $\mathrm{R}$, medium $\mathrm{M}$, and left L); $\geq 100 \mathrm{~kb}$ for clustering (I, II, III, IV, etc.), and $\geq 10 \mathrm{~kb}$ for sub-clustering (a, b, c, d, etc.). See Table 1.

The phylogenetic clustering was defined by constructing a phylogenetic tree with the promoter regions and coding regions of the functional genes and pseudogenes. Each phylogenetic clustering was defined according to the clades with more than 5 genes, and numbered as follows: "clustering $\mathrm{C} 1, \mathrm{C} 2, \mathrm{C} 3$, etc." The reconstruction of the phylogenetic tree was built using a neighbor-joining method with a bootstrapping value of 1000 . The MEGA 4.0 software was downloaded from www.megasoftware.net (Tamura et al., 2007) and used for all phylogenetic analyses.

Finally, chromosomal and phylogenetic clustering was related to the conserved regions in the promoter regions. 


\begin{tabular}{|c|c|c|c|c|c|c|c|c|c|}
\hline Name & Name according to ID & $\mathrm{Chr}$ & Start & End & Strand & HCR region & Type & Size & $\begin{array}{c}\text { Intergenic } \\
\text { distance (ID) }\end{array}$ \\
\hline OR4G4P & $1 \mathrm{R}$ & $\operatorname{chr} 1$ & 41316 & 43258 & $(+)$ & HCR II & Pseudogene & 1942 & first \\
\hline OR13Z2P & $1 \mathrm{MI}$ & $\operatorname{chr} 1$ & 145382936 & 145384490 & $(+)$ & HCR II & Pseudogene & 1554 & 145339678 \\
\hline OR10K1 & $1 \mathrm{MII}$ & $\operatorname{chr} 1$ & 156700977 & 156702914 & $(+)$ & HCR I & Functional & 1937 & 11316487 \\
\hline OR10R3P & $1 \mathrm{MII}$ & $\operatorname{chr} 1$ & 156726634 & 156728571 & $(+)$ & HCR II & Pseudogene & 1937 & 23720 \\
\hline OR10AE1P & $1 \mathrm{MIII}$ & $\operatorname{chr} 1$ & 157818038 & 157819634 & $(-)$ & HCR II & Pseudogene & 1596 & 1089467 \\
\hline OR9H1P & $1 \mathrm{La}$ & $\operatorname{chr} 1$ & 246003836 & 246005758 & $(+)$ & HCR II & Pseudogene & 1922 & 88184202 \\
\hline OR5AT1 & $1 \mathrm{La}$ & $\operatorname{chr} 1$ & 246044729 & 246046654 & $(-)$ & HCR I & Functional & 1925 & 38971 \\
\hline OR11L1 & $1 \mathrm{La}$ & $\operatorname{chr} 1$ & 246070857 & 246072821 & $(-)$ & HCR I & Functional & 1964 & 24203 \\
\hline OR2L8 & $1 \mathrm{Lb}$ & $\operatorname{chr} 1$ & 246177784 & 246179718 & $(+)$ & HCR II & Functional & 1934 & 104963 \\
\hline OR2AK2 & $1 \mathrm{Lb}$ & $\operatorname{chr} 1$ & 246194303 & 246196261 & $(+)$ & HCR I & Functional & 1958 & 14585 \\
\hline OR2L1P & $1 \mathrm{Lb}$ & chr1 & 246219193 & 246221116 & $(+)$ & HCR I & Pseudogene & 1923 & 22932 \\
\hline OR2AS2P & $1 \mathrm{Lc}$ & $\operatorname{chr} 1$ & 246727103 & 246728583 & $(+)$ & HCR II & Pseudogene & 1480 & 505987 \\
\hline OR7E46P & $2 \mathrm{R}$ & chr2 & 71117360 & 71119303 & $(+)$ & HCR II & Pseudogene & 1943 & first \\
\hline OR7E62P & $2 \mathrm{R}$ & chr2 & 71134777 & 71136796 & $(+)$ & HCR I & Pseudogene & 2019 & 15474 \\
\hline OR7E102P & $2 \mathrm{~L}$ & chr2 & 95575054 & 95577048 & $(+)$ & HCR II & Pseudogene & 1994 & 24438258 \\
\hline OR7E122P & $3 R$ & chr3 & 8703921 & 8705840 & $(+)$ & HCR I & Pseudogene & 1919 & first \\
\hline OR7E66P & $3 \mathrm{Ma}$ & $\operatorname{chr} 3$ & 75479689 & 75481604 & $(-)$ & HCR I & Pseudogene & 1915 & 66773849 \\
\hline OR7E22P & $3 \mathrm{Ma}$ & $\operatorname{chr} 3$ & 75488327 & 75490351 & $(-)$ & HCR I & Pseudogene & 2024 & 6723 \\
\hline OR7E55P & $3 \mathrm{Ma}$ & chr3 & 75502255 & 75504204 & $(-)$ & HCR I & Pseudogene & 1949 & 11904 \\
\hline OR7E121P & $3 \mathrm{Mb}$ & $\operatorname{chr} 3$ & 75730493 & 75732514 & $(-)$ & HCR I & Pseudogene & 2021 & 226289 \\
\hline OR7E100P & $3 \mathrm{LI}$ & $\operatorname{chr} 3$ & 113725724 & 113727737 & $(-)$ & HCR I & Pseudogene & 2013 & 37993210 \\
\hline OR7E130P & 3LII & chr3 & 126903910 & 126905871 & $(+)$ & HCR I & Pseudogene & 1961 & 13176173 \\
\hline OR7E29P & $3 \mathrm{LII}$ & chr3 & 126912660 & 126914603 & $(+)$ & HCR I & Pseudogene & 1943 & 6789 \\
\hline OR7E93P & $3 \mathrm{LII}$ & $\operatorname{chr} 3$ & 126925014 & 126927047 & $(+)$ & HCR I & Pseudogene & 2033 & 10411 \\
\hline OR7E53P & $3 \mathrm{LII}$ & $\operatorname{chr} 3$ & 126934770 & 126936777 & $(+)$ & HCR I & Pseudogene & 2007 & 7723 \\
\hline OR7E97P & 3LII & chr3 & 126947609 & 126949642 & $(+)$ & HCR I & Pseudogene & 2033 & 10832 \\
\hline OR7E129P & 3LIII & $\operatorname{chr} 3$ & 131223090 & 131225113 & $(-)$ & HCR I & Pseudogene & 2023 & 4273448 \\
\hline OR7E21P & 3LIII & $\operatorname{chr} 3$ & 131236023 & 131238075 & $(-)$ & HCR I & Pseudogene & 2052 & 10910 \\
\hline OR7E99P & $4 \mathrm{I}$ & $\operatorname{chr} 4$ & 4209108 & 4211131 & $(-)$ & HCR I & Pseudogene & 2023 & first \\
\hline OR7E43P & $4 \mathrm{I}$ & chr4 & 4226950 & 4228918 & $(-)$ & HCR II & Pseudogene & 1968 & 15819 \\
\hline OR7E85P & $4 \mathrm{II}$ & $\operatorname{chr} 4$ & 9093449 & 9095475 & $(+)$ & HCR I & Pseudogene & 2026 & 4864531 \\
\hline OR2V1 & 5 & chr5 & 180483967 & 180485910 & $(-)$ & HCR I & Functional & 1943 & first \\
\hline OR2V2 & 5 & $\operatorname{chr} 5$ & 180513550 & 180515493 & $(+)$ & HCR II & Functional & 1943 & 27640 \\
\hline OR2B2 & $6 \mathrm{RI}$ & chr6 & 27987007 & 27989076 & $(-)$ & HCR II & Functional & 2069 & first \\
\hline OR2W6P & $6 \mathrm{RI}$ & chr6 & 28012161 & 28014157 & $(+)$ & HCR I & Pseudogene & 1996 & 23085 \\
\hline OR12D2 & 6RII & chr6 & 29471457 & 29473376 & $(+)$ & HCR I & Functional & 1919 & 1457300 \\
\hline OR12D1P & 6RII & chr6 & 29492037 & 29493964 & $(+)$ & HCR II & Pseudogene & 1927 & 18661 \\
\hline OR11A1 & 6RII & chr6 & 29502454 & 29504397 & $(-)$ & HCR I & Functional & 1943 & 8490 \\
\hline OR2A4 & $6 \mathrm{~L}$ & chr6 & 132063306 & 132065234 & $(-)$ & HCR I & Functional & 1928 & 102558909 \\
\hline OR10AH1P & $7 \mathrm{R}$ & $\operatorname{chr} 7$ & 5122247 & 5124244 & $(+)$ & HCR I & Pseudogene & 1997 & first \\
\hline OR9A3P & $7 \mathrm{LI}$ & chr7 & 141208130 & 141210088 & $(+)$ & HCR I & Pseudogene & 1958 & 136083886 \\
\hline OR9A4 & $7 \mathrm{LI}$ & $\operatorname{chr} 7$ & 141264146 & 141266086 & $(+)$ & HCR I & Functional & 1940 & 54058 \\
\hline OR6V1 & 7LIIa & chr7 & 142458561 & 142460498 & $(+)$ & HCR I & Functional & 1937 & 1192475 \\
\hline OR2A41P & $7 \mathrm{LIIb}$ & $\operatorname{chr} 7$ & 143404420 & 143405880 & $(+)$ & HCR I & Pseudogene & 1460 & 943922 \\
\hline OR2A7 & 7LIIc & chr7 & 143586726 & 143588654 & $(-)$ & HCR I & Functional & 1928 & 180846 \\
\hline OR7E158P & $8 \mathrm{a}$ & chr8 & 11814815 & 11816713 & $(-)$ & HCR I & Pseudogene & 1898 & first \\
\hline OR7E161P & $8 \mathrm{a}$ & chr8 & 11823487 & 11825481 & $(-)$ & HCR I & Pseudogene & 1994 & 6774 \\
\hline OR7E160P & $8 b$ & chr8 & 11928536 & 11930560 & $(-)$ & HCR I & Pseudogene & 2024 & 103055 \\
\hline OR7E8P & $8 \mathrm{c}$ & chr8 & 12586021 & 12588045 & $(-)$ & HCR I & Pseudogene & 2024 & 655461 \\
\hline OR7E15P & $8 \mathrm{c}$ & chr8 & 12598175 & 12600144 & $(-)$ & HCR I & Pseudogene & 1969 & 10130 \\
\hline OR7E10P & $8 \mathrm{c}$ & chr8 & 12604933 & 12606918 & $(-)$ & HCR I & Pseudogene & 1985 & 4789 \\
\hline OR13C6P & $9 \mathrm{R}$ & chr9 & 35981337 & 35983282 & $(-)$ & HCR II & Pseudogene & 1945 & first \\
\hline OR2AM1P & $9 \mathrm{R}$ & chr9 & 36010709 & 36012112 & $(+)$ & HCR I & Pseudogene & 1403 & 27427 \\
\hline OR7E116P & 9LI & chr9 & 92033201 & 92035225 & $(+)$ & HCR I & Pseudogene & 2024 & 56021089 \\
\hline OR13C8 & 9LII & chr9 & 106370271 & 106372229 & $(+)$ & HCR II & Functional & 1958 & 14335046 \\
\hline OR2K2 & 9LIII & chr9 & 113129588 & 113131534 & $(-)$ & HCR I & Functional & 1946 & 6757359 \\
\hline
\end{tabular}

Continued on next page 
Human olfactory receptor genes: promoter region description

Table 1. Continued.

\begin{tabular}{|c|c|c|c|c|c|c|c|c|c|}
\hline Name & Name according to ID & $\mathrm{Chr}$ & Start & End & Strand & HCR region & Type & Size & $\begin{array}{c}\text { Intergenic } \\
\text { distance (ID) }\end{array}$ \\
\hline OR1L1 & 9LIV & chr9 & 124462817 & 124464745 & $(+)$ & HCR II & Functional & 1928 & 11331283 \\
\hline OR1L3 & 9LIV & chr9 & 124476231 & 124478201 & $(+)$ & HCR I & Functional & 1970 & 11486 \\
\hline OR1L6 & 9LIV & chr9 & 124550949 & 124552880 & $(+)$ & HCR II & Functional & 1931 & 72748 \\
\hline OR5C1 & 9LIV & chr9 & 124590034 & 124591992 & $(+)$ & HCR II & Functional & 1958 & 37154 \\
\hline OR1K1 & 9LIV & $\operatorname{chr} 9$ & 124601224 & 124603170 & $(+)$ & HCR II & Functional & 1946 & 9232 \\
\hline OR7E110P & $10 \mathrm{R}$ & $\operatorname{chr} 10$ & 15068882 & 15070476 & $(-)$ & HCR I & Pseudogene & 1594 & first \\
\hline OR7E26P & $10 \mathrm{R}$ & $\operatorname{chr} 10$ & 15081109 & 15083090 & $(-)$ & HCR I & Pseudogene & 1981 & 10633 \\
\hline OR7E115P & $10 \mathrm{R}$ & $\operatorname{chr} 10$ & 15089878 & 15091884 & $(-)$ & HCR I & Pseudogene & 2006 & 6788 \\
\hline OR13A1 & $10 \mathrm{~L}$ & $\operatorname{chr} 10$ & 45118894 & 45120819 & $(-)$ & HCR I & Functional & 1925 & 30027010 \\
\hline OR7E12P & 11RIa & chr11 & 3368617 & 3370555 & $(-)$ & HCR I & Pseudogene & 1938 & first \\
\hline OR7E117P & $11 \mathrm{RIb}$ & $\operatorname{chr} 11$ & 3577451 & 3579475 & $(-)$ & HCR I & Pseudogene & 2024 & 206896 \\
\hline OR51D1 & 11RIIa & chr11 & 4616598 & 4618568 & $(+)$ & HCR I & Functional & 1970 & 1037123 \\
\hline OR51A9P & 11RIIa & chr11 & 4638639 & 4640536 & $(-)$ & HCR II & Pseudogene & 1897 & 20071 \\
\hline OR51F3P & 11RIIa & $\operatorname{chr} 11$ & 4713985 & 4715884 & $(-)$ & HCR I & Pseudogene & 1899 & 73449 \\
\hline OR51F1 & 11RIIa & $\operatorname{chr} 11$ & 4746789 & 4748723 & $(-)$ & HCR I & Functional & 1934 & 30905 \\
\hline OR52R1 & 11RIIa & $\operatorname{chr} 11$ & 4781243 & 4783186 & $(-)$ & HCR I & Functional & 1943 & 32520 \\
\hline OR51A7 & 11RIIb & $\operatorname{chr} 11$ & 4884177 & 4886111 & $(+)$ & HCR II & Functional & 1934 & 100991 \\
\hline OR51P1P & 11RIIc & $\operatorname{chr} 11$ & 4991945 & 4993882 & $(+)$ & HCR II & Pseudogene & 1937 & 105834 \\
\hline OR52A5 & 11RIId & $\operatorname{chr} 11$ & 5109502 & 5111448 & $(-)$ & HCR I & Functional & 1946 & 115620 \\
\hline OR52Z1P & 11RIId & $\operatorname{chr} 11$ & 5155527 & 5157416 & $(-)$ & HCR I & Pseudogene & 1889 & 44079 \\
\hline OR51J1 & 11RIIe & $\operatorname{chr} 11$ & 5379404 & 5381350 & $(+)$ & HCR I & Functional & 1946 & 221988 \\
\hline OR52B6 & 11RIIf & $\operatorname{chr} 11$ & 5557747 & 5559687 & $(+)$ & HCR I & Functional & 1940 & 176397 \\
\hline OR52N4 & 11RIIg & $\operatorname{chr} 11$ & 5731548 & 5733509 & $(+)$ & HCR II & Functional & 1961 & 171861 \\
\hline OR56B4 & 11RIIh & $\operatorname{chr} 11$ & 6084586 & 6086541 & $(+)$ & HCR I & Functional & 1955 & 351077 \\
\hline OR4X2 & $11 \mathrm{MIa}$ & $\operatorname{chr} 11$ & 48222233 & 48224140 & $(+)$ & HCR II & Functional & 1907 & 42135692 \\
\hline OR4C5 & $11 \mathrm{MIb}$ & $\operatorname{chr} 11$ & 48343617 & 48345593 & $(-)$ & HCR II & Functional & 1976 & 119477 \\
\hline OR4C10P & $11 \mathrm{MIb}$ & $\operatorname{chr} 11$ & 48410349 & 48412301 & $(-)$ & HCR I & Pseudogene & 1952 & 64756 \\
\hline OR4C46 & 11MII & $\operatorname{chr} 11$ & 51370859 & 51372784 & $(+)$ & HCR I & Functional & 1925 & 2958558 \\
\hline OR7E5P & $11 \mathrm{MIIIa}$ & $\operatorname{chr} 11$ & 55503167 & 55505158 & $(-)$ & HCR I & Pseudogene & 1991 & 4130383 \\
\hline $\mathrm{OR} 8 \mathrm{H} 2$ & $11 \mathrm{MIIIb}$ & $\operatorname{chr} 11$ & 55628096 & 55630030 & $(+)$ & HCR I & Functional & 1934 & 122938 \\
\hline OR8H3 & $11 \mathrm{MIIIb}$ & $\operatorname{chr} 11$ & 55645426 & 55647360 & $(+)$ & HCR I & Functional & 1934 & 15396 \\
\hline OR8J3 & $11 \mathrm{MIIIb}$ & $\operatorname{chr} 11$ & 55660827 & 55662770 & $(-)$ & HCR I & Functional & 1943 & 13467 \\
\hline OR5M8 & $11 \mathrm{MIIIc}$ & $\operatorname{chr} 11$ & 56014491 & 56016422 & $(-)$ & HCR I & Functional & 1931 & 351721 \\
\hline OR5M10 & $11 \mathrm{MIIIc}$ & $\operatorname{chr} 11$ & 56100830 & 56102773 & $(-)$ & HCR I & Functional & 1943 & 84408 \\
\hline OR5AP2 & $11 \mathrm{MIIIc}$ & $\operatorname{chr} 11$ & 56165545 & 56167494 & $(-)$ & HCR I & Functional & 1949 & 62772 \\
\hline OR9G4 & 11MIIId & $\operatorname{chr} 11$ & 56266884 & 56268818 & $(-)$ & HCR II & Functional & 1934 & 99390 \\
\hline OR5BQ1P & $11 \mathrm{MIII}$ & $\operatorname{chr} 11$ & 56552439 & 56553773 & $(+)$ & HCR II & Pseudogene & 1334 & 283621 \\
\hline OR5AZ1P & $11 \mathrm{MIIIf}$ & $\operatorname{chr} 11$ & 57441351 & 57443278 & $(-)$ & HCR I & Pseudogene & 1927 & 887578 \\
\hline OR5BD1P & $11 \mathrm{MIIIf}$ & $\operatorname{chr} 11$ & 57469604 & 57471519 & $(-)$ & HCR II & Pseudogene & 1915 & 26326 \\
\hline OR9Q1 & $11 \mathrm{MIIIg}$ & $\operatorname{chr} 11$ & 57702494 & 57704422 & $(+)$ & HCR I & Functional & 1928 & 230975 \\
\hline OR10Q1 & $11 \mathrm{MIIIg}$ & $\operatorname{chr} 11$ & 57751968 & 57753923 & $(-)$ & HCR II & Functional & 1955 & 47546 \\
\hline OR10Q2P & $11 \mathrm{MIIIg}$ & chr11 & 57815878 & 57817819 & $(-)$ & HCR II & Pseudogene & 1941 & 61955 \\
\hline OR5AN1 & $11 \mathrm{MIVa}$ & $\operatorname{chr} 11$ & 58887509 & 58889440 & $(+)$ & HCR I & Functional & 1931 & 1069690 \\
\hline OR4D11 & $11 \mathrm{MIVb}$ & $\operatorname{chr} 11$ & 59026626 & 59028557 & $(+)$ & HCR II & Functional & 1931 & 137186 \\
\hline OR4D9 & $11 \mathrm{MIVb}$ & $\operatorname{chr} 11$ & 59037963 & 59039903 & $(+)$ & HCR II & Functional & 1940 & 9406 \\
\hline OR4D7P & $11 \mathrm{MIVb}$ & $\operatorname{chr} 11$ & 59054747 & 59056759 & $(+)$ & HCR II & Pseudogene & 2012 & 14844 \\
\hline OR10V1 & $11 \mathrm{MIVc}$ & chr11 & 59236969 & 59238894 & $(-)$ & HCR II & Functional & 1925 & 180210 \\
\hline OR7E145P & $11 \mathrm{MVa}$ & $\operatorname{chr} 11$ & 67246507 & 67248531 & $(-)$ & HCR I & Pseudogene & 2024 & 8007613 \\
\hline OR7E11P & $11 \mathrm{MVa}$ & $\operatorname{chr} 11$ & 67259645 & 67261594 & $(-)$ & HCR I & Pseudogene & 1949 & 11114 \\
\hline OR7E1P & $11 \mathrm{MVb}$ & $\operatorname{chr} 11$ & 67498283 & 67500304 & $(-)$ & HCR I & Pseudogene & 2021 & 236689 \\
\hline OR7E87P & 11MVIa & $\operatorname{chr} 11$ & 70981089 & 70983107 & $(+)$ & HCR I & Pseudogene & 2018 & 3480785 \\
\hline OR7E4P & 11MVIa & $\operatorname{chr} 11$ & 71007708 & 71009726 & $(+)$ & HCR I & Pseudogene & 2018 & 24601 \\
\hline OR7E128P & $11 \mathrm{MVIb}$ & $\operatorname{chr} 11$ & 71281102 & 71283132 & $(+)$ & HCR I & Pseudogene & 2030 & 271376 \\
\hline OR7E126P & $11 \mathrm{MVIb}$ & $\operatorname{chr} 11$ & 71290880 & 71292813 & $(+)$ & HCR I & Pseudogene & 1933 & 7748 \\
\hline OR6M2P & $11 \mathrm{La}$ & $\operatorname{chr} 11$ & 123216877 & 123218821 & $(-)$ & HCR I & Pseudogene & 1944 & 51924064 \\
\hline OR4D5 & $11 \mathrm{Lb}$ & $\operatorname{chr} 11$ & 123314535 & 123316487 & $(+)$ & HCR I & Functional & 1952 & 95714 \\
\hline OR10D5P & $11 \mathrm{Lc}$ & $\operatorname{chr} 11$ & 123429711 & 123431643 & $(+)$ & HCR I & Pseudogene & 1932 & 113224 \\
\hline OR8B3 & $11 \mathrm{Ld}$ & $\operatorname{chr} 11$ & 123771520 & 123773457 & $(-)$ & HCR II & Functional & 1937 & 339877 \\
\hline
\end{tabular}

Continued on next page 
Table 1. Continued.

\begin{tabular}{|c|c|c|c|c|c|c|c|c|c|}
\hline Name & Name according to ID & $\mathrm{Chr}$ & Start & End & Strand & HCR region & Type & Size & $\begin{array}{c}\text { Intergenic } \\
\text { distance (ID) }\end{array}$ \\
\hline OR8A2P & $11 \mathrm{Ld}$ & $\operatorname{chr} 11$ & 123834671 & 123836632 & $(+)$ & HCR II & Pseudogene & 1961 & 61214 \\
\hline OR7E148P & $12 \mathrm{R}$ & $\operatorname{chr} 12$ & 8471526 & 8473477 & $(-)$ & HCR I & Pseudogene & 1951 & first \\
\hline OR7E149P & $12 \mathrm{R}$ & $\operatorname{chr} 12$ & 8481267 & 8483246 & $(-)$ & HCR I & Pseudogene & 1979 & 7790 \\
\hline OR5BT1P & $12 \mathrm{LIa}$ & $\operatorname{chr} 12$ & 47065402 & 47067355 & $(-)$ & HCR I & Pseudogene & 1953 & 38582156 \\
\hline OR8S1 & $12 \mathrm{LIb}$ & $\operatorname{chr} 12$ & 47204683 & 47206617 & $(+)$ & HCR I & Functional & 1934 & 137328 \\
\hline OR5BS1P & $12 \mathrm{LIb}$ & chr12 & 47238933 & 47240864 & $(+)$ & HCR II & Pseudogene & 1931 & 32316 \\
\hline OR7E47P & $12 \mathrm{LII}$ & $\operatorname{chr} 12$ & 50786370 & 50788314 & $(+)$ & HCR I & Pseudogene & 1944 & 3545506 \\
\hline OR6C74 & $12 \mathrm{LIII}$ & $\operatorname{chr} 12$ & 53926340 & 53928274 & $(+)$ & HCR I & Functional & 1934 & 3138026 \\
\hline OR6C1 & 12LIII & $\operatorname{chr} 12$ & 53999652 & 54001586 & $(+)$ & HCR II & Functional & 1934 & 71378 \\
\hline OR6C75 & 12LIII & $\operatorname{chr} 12$ & 54044163 & 54046097 & $(+)$ & HCR I & Functional & 1934 & 42577 \\
\hline OR6C65 & 12LIII & $\operatorname{chr} 12$ & 54079581 & 54081515 & $(+)$ & HCR I & Functional & 1934 & 33484 \\
\hline OR7E36P & $13 \mathrm{R}$ & $\operatorname{chr} 13$ & 40903401 & 40905394 & $(-)$ & HCR I & Pseudogene & 1993 & first \\
\hline OR7E155P & $13 R$ & $\operatorname{chr} 13$ & 40911974 & 40913998 & $(-)$ & HCR I & Pseudogene & 2024 & 6580 \\
\hline OR7E111P & $13 \mathrm{~L}$ & $\operatorname{chr} 13$ & 67373378 & 67375357 & $(+)$ & HCR I & Pseudogene & 1979 & 26459380 \\
\hline OR7E33P & $13 \mathrm{~L}$ & $\operatorname{chr} 13$ & 67382135 & 67384081 & $(+)$ & HCR I & Pseudogene & 1946 & 6778 \\
\hline OR11H2 & $14 \mathrm{Ra}$ & $\operatorname{chr} 14$ & 19250939 & 19252882 & $(-)$ & HCR I & Functional & 1943 & first \\
\hline OR4K3P & $14 \mathrm{Rb}$ & $\operatorname{chr} 14$ & 19406200 & 19408142 & $(-)$ & HCR I & Pseudogene & 1942 & 153318 \\
\hline OR4K1 & $14 \mathrm{Rb}$ & $\operatorname{chr} 14$ & 19472667 & 19474598 & $(+)$ & HCR I & Functional & 1931 & 64525 \\
\hline OR4K17 & $14 \mathrm{Rc}$ & chr14 & 19654500 & 19656434 & $(+)$ & HCR I & Functional & 1934 & 179902 \\
\hline OR11H5P & $14 \mathrm{Rc}$ & chr14 & 19746297 & 19748194 & $(+)$ & HCR II & Pseudogene & 1897 & 89863 \\
\hline OR7E105P & $14 \mathrm{~L}$ & chr14 & 51292459 & 51294453 & $(+)$ & HCR I & Pseudogene & 1994 & 31544265 \\
\hline OR4G6P & 15 & $\operatorname{chr} 15$ & 100295277 & 100297219 & $(-)$ & HCR II & Pseudogene & 1942 & unique \\
\hline OR2C1 & 16 & $\operatorname{chr} 16$ & 3344943 & 3346877 & $(+)$ & HCR II & Functional & 1934 & unique \\
\hline OR1P1P & $17 \mathrm{a}$ & $\operatorname{chr} 17$ & 3003938 & 3005896 & $(-)$ & HCR I & Pseudogene & 1958 & first \\
\hline OR1R1P & $17 b$ & $\operatorname{chr} 17$ & 3234975 & 3236915 & $(+)$ & HCR II & Pseudogene & 1940 & 229079 \\
\hline OR1E1 & $17 \mathrm{~b}$ & $\operatorname{chr} 17$ & 3247514 & 3249454 & $(-)$ & HCR I & Functional & 1940 & 10599 \\
\hline OR3A3 & $17 \mathrm{~b}$ & $\operatorname{chr} 17$ & 3269631 & 3271574 & $(+)$ & HCR I & Functional & 1943 & 20177 \\
\hline OR1E2 & $17 \mathrm{~b}$ & $\operatorname{chr} 17$ & 3282918 & 3284885 & $(-)$ & HCR II & Functional & 1967 & 11344 \\
\hline OR4G3P & 19I & chr19 & 44063 & 46005 & $(+)$ & HCR II & Pseudogene & 1942 & first \\
\hline OR4F17 & 19I & chr19 & 60680 & 62593 & $(+)$ & HCR I & Functional & 1913 & 14675 \\
\hline OR2Z1 & 19IIa & chr19 & 8701392 & 8703332 & $(+)$ & HCR I & Functional & 1940 & 8638799 \\
\hline OR1M4P & $19 \mathrm{IIb}$ & chr19 & 9054613 & 9056074 & $(-)$ & HCR I & Pseudogene & 1461 & 351281 \\
\hline OR1M1 & $19 \mathrm{IIb}$ & $\operatorname{chr} 19$ & 9063922 & 9065859 & $(+)$ & HCR II & Functional & 1937 & 7848 \\
\hline OR7G2 & $19 I I b$ & chr19 & 9073949 & 9075919 & $(-)$ & HCR II & Functional & 1970 & 8090 \\
\hline OR7G1 & $19 \mathrm{IIb}$ & chr19 & 9086508 & 9088439 & $(-)$ & HCR II & Functional & 1931 & 10589 \\
\hline OR7G15P & $19 \mathrm{IIb}$ & chr19 & 9093761 & 9095163 & $(-)$ & HCR II & Pseudogene & 1402 & 5322 \\
\hline OR7D2 & $19 \mathrm{IIb}$ & $\operatorname{chr} 19$ & 9156459 & 9158393 & $(+)$ & HCR I & Functional & 1934 & 61296 \\
\hline OR7E25P & $19 \mathrm{IIb}$ & chr19 & 9174929 & 9176908 & $(+)$ & HCR I & Pseudogene & 1979 & 16536 \\
\hline OR7D4 & $19 \mathrm{IIb}$ & chr19 & 9185579 & 9187513 & $(-)$ & HCR I & Functional & 1934 & 8671 \\
\hline OR7E24 & $19 \mathrm{IIb}$ & chr19 & 9221721 & 9223736 & $(+)$ & HCR I & Functional & 2015 & 34208 \\
\hline OR7C1 & 19IIIa & chr19 & 14770990 & 14772948 & $(-)$ & HCR I & Functional & 1958 & 5547254 \\
\hline OR7A5 & 19IIIa & chr19 & 14799098 & 14801053 & $(-)$ & HCR I & Functional & 1955 & 26150 \\
\hline OR7A10 & 19IIIa & chr19 & 14812764 & 14814689 & $(-)$ & HCR II & Functional & 1925 & 11711 \\
\hline OR7A11P & 19IIIa & chr19 & 14887196 & 14889213 & $(+)$ & HCR I & Pseudogene & 2017 & 72507 \\
\hline OR7C2 & 19IIIa & chr19 & 14912302 & 14914257 & $(+)$ & HCR II & Functional & 1955 & 23089 \\
\hline OR1I1 & 19IIIb & chr19 & 15057878 & 15059821 & $(+)$ & HCR I & Functional & 1943 & 143621 \\
\hline OR10H3 & 19IIIc & chr19 & 15712204 & 15714150 & $(+)$ & HCR II & Functional & 1946 & 652383 \\
\hline OR10H5 & 19IIIc & chr19 & 15764860 & 15766803 & $(+)$ & HCR II & Functional & 1943 & 50710 \\
\hline OR10H1 & 19IIIc & chr19 & 15778895 & 15780847 & $(-)$ & HCR I & Functional & 1952 & 12092 \\
\hline OR1AB1P & 19IIId & chr19 & 16022788 & 16024525 & $(+)$ & HCR II & Pseudogene & 1737 & 241941 \\
\hline
\end{tabular}

\section{RESULTS}

\section{hOR functional genes and pseudogenes}

A total of 851 hOR gene sequences were downloaded from the HORDE database, from which 389 are functional genes and 462 are pseudogenes. These values correspond to 
those found in known databases and previously reported studies (Zozulya et al., 2001; Glusman et al., 2001; Malnic et al., 2004; Niimura and Nei, 2003).

\section{Regularities in the promoter regions of the hOR genes}

Our approach resulted in the discovery of two highly conserved regions (HCR) upstream from the TSS. We designated these regions HCR I and HCR II for 111 and 53 genes, respectively. They represent a structural model of gene regulation for the hOR gene (Figure 1). This number of genes represents $19 \%$ of the hOR gene family, and each HCR is present in several functional genes and pseudogenes. Each HCR has different structural characteristics as shown in Table 2.

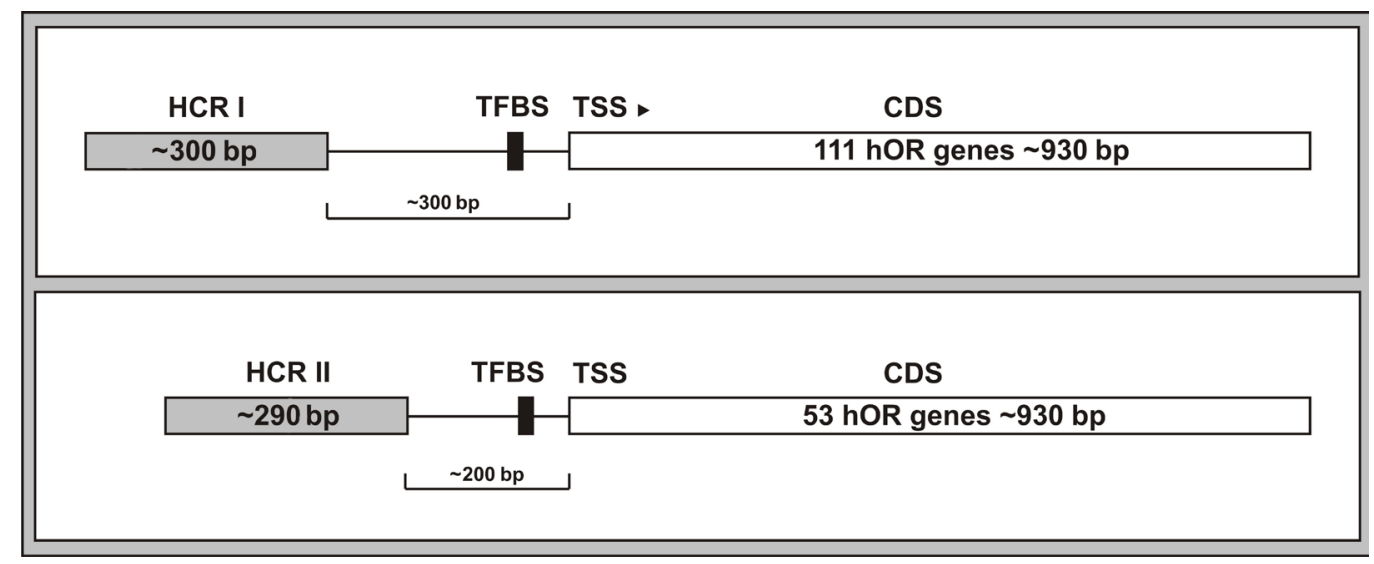

Figure 1. Structural model of gene regulation for the human olfactory gene family. Structural model of human olfactory receptor (hOR) genes with the highly conserved regions (HCR I and HCR II). The blocks represent the structure of the gene with its average length. Each gene has one HCR and a possible transcription factor binding site (TFBS) upstream from the transcription start sites (TSS).

\begin{tabular}{lccccc}
\multicolumn{6}{l}{ Table 2. Structural characteristics of the highly conserved regions, HCR I and HCR II. } \\
\hline $\begin{array}{c}\text { Number of } \\
\text { Region }\end{array}$ & $\begin{array}{c}\text { Number of } \\
\text { pseudogenes }\end{array}$ & $\begin{array}{c}\text { Percentage of } \\
\text { similarity }\end{array}$ & HCR length & $\begin{array}{c}\text { Distance } \\
\text { HCR-TSS }\end{array}$ \\
\hline HCR I & 49 & 62 & $\approx 75 \%$ & $\approx 300 \mathrm{bp}$ & $\approx 300 \mathrm{bp}$ \\
HCR II & 28 & 25 & $\approx 70 \%$ & $\approx 290 \mathrm{bp}$ & $\approx 200 \mathrm{bp}$ \\
\hline
\end{tabular}

TSS $=$ transcription start sites.

To confirm that these regions are not part of a possible exon in an interrupted gene, we conducted an exploratorium search of the hOR receptor interrupted genes using the gbk file from GenBank (Human, May 2004 - hg 17, NCBI Build 35). We found that only 5 genes (OR3A2, OR52E5, OR56A3, OR7G1, and OR8S1) are not single-exon.

The BLAST search on the TRRD (Kolchanov et al., 2002) allowed for the discovery of many different and conserved motifs in each HCR, as shown in Figure 2. However, the most important motifs were predicted via the weeder prediction tool (Pavesi et al., 2004). These motifs represent O/E-like and homeodomain binding sites in about $90 \%$ of genes with an HCR. It is noteworthy that the HCR II motifs were also defined in the olfactory receptor genes in mice and represent possible negative control function of the gene (Michaloski et al., 2006; Hoppe et al., 2006). 


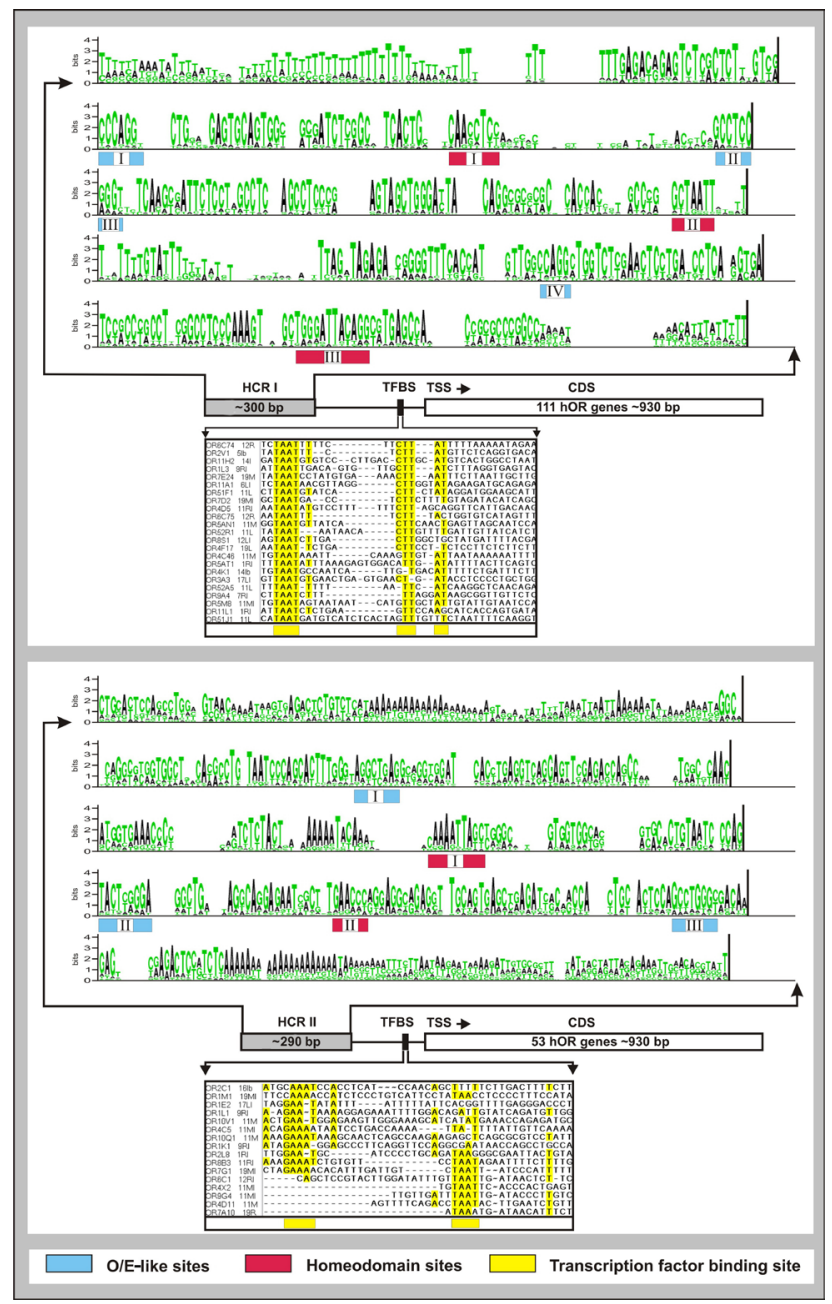

Figure 2. Highly conserved regions (HCR). Highly conserved regions with the most representative motifs and the most conserved transcription factors in some genes. TFBS $=$ transcription factor binding sites; TSS $=$ transcription start sites.

Michaloski et al. (2006) associated the O/E-like and homeodomain motifs with a possible enhancer function, as witnessed herein by comparing the HCR sequences with TRRD. This possible function was validated by looking for particular transcription factor sites for each HCR, $-100 \mathrm{bp}$ from the TSS of each gene. We used the Hctata Tool (http://zeus2.itb.cnr.it/ webgene/ wwwHC tata.html) to predict any common TATA-box in the sequence. A multiple alignment using the T-Coffee tool, and visual inspection then permitted the definition of the transcription factor binding sites in the sequences. As a result of this process, different TATA-box and transcription factor binding sites were found in different groups of genes with HCR I and HCR II, as shown in Figure 2. The number of genes with these transcriptional factor binding sites is about $46 \%$ for HCR I and 35\% for HCR II. These results indicate that the HCR may act on different transcription factors and in different positions, as previously reported in mice by Michaloski et al. (2006). 


\section{Chromosomal and phylogenetic clustering analyses}

The cluster distribution we established was similar to that previously reported for the complete set of hOR genes (Zozulya et al., 2001; Glusman et al., 2001; Niimura and Nei, 2003). We defined 33 chromosomal clusterings, as shown in Figure 3A. Gene distribution in the regions is not a particular characteristic for chromosomes 4, 5, 8, 17, and 19; however, the distribution in sub-clusters is found on chromosomes $1,3,7,8,11,12,14,17$, and 19. Chromosome 11 has the largest number of genes with an HCR, and these genes are distributed in many clusters and sub-clusters mainly in the $\mathrm{R}$ and $\mathrm{M}$ regions of the chromosome.

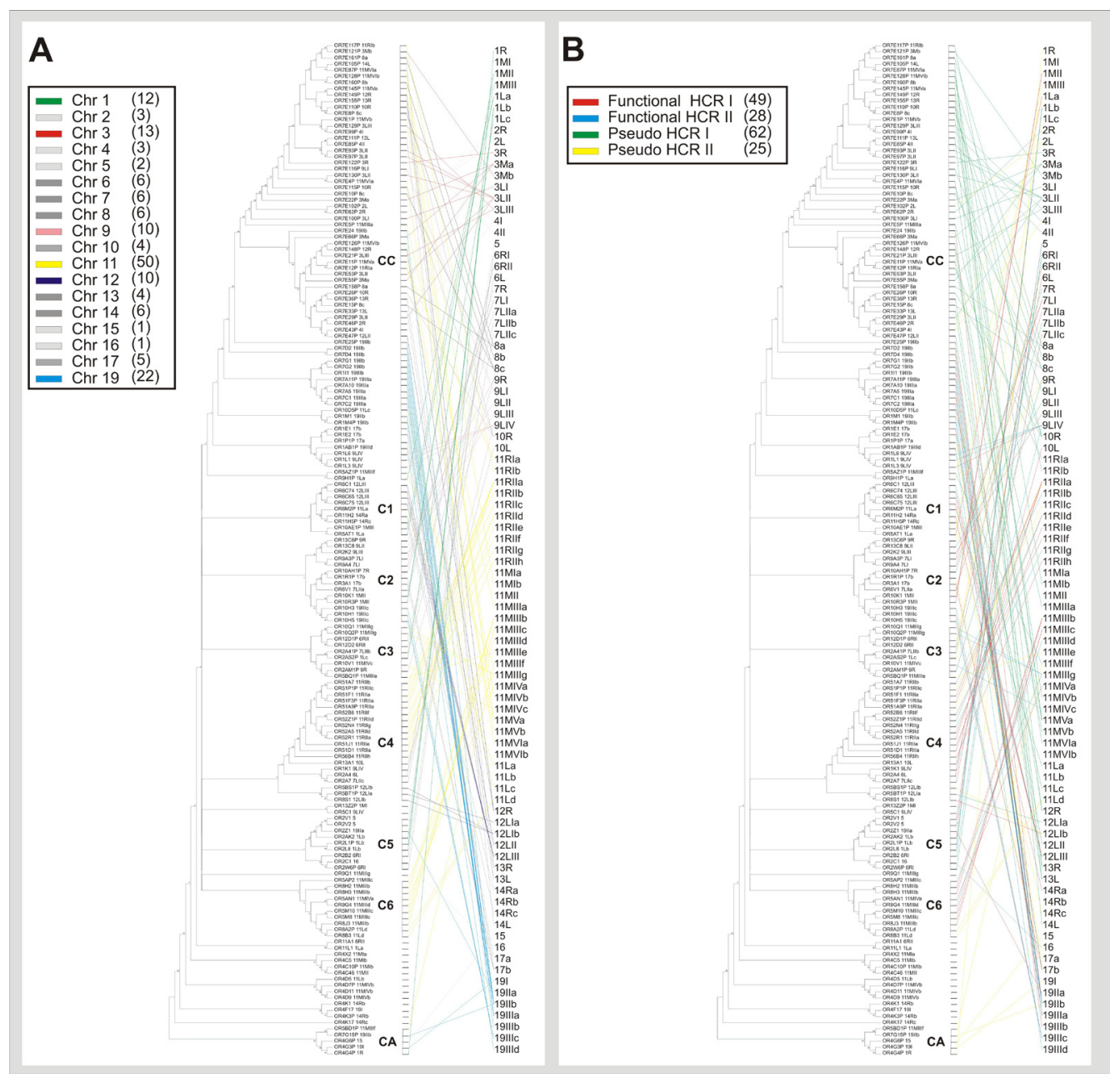

Figure 3. Relationship between phylogenetic and chromosomal clustering and the higly conserved regions (HCR). Phylogenetic tree reconstructed using a neighbor-joining method with a bootstrapping value of 1000 . A. Phylogenetic clustering and chromosomal clustering of the genes are joined by lines with different colors by each chromosome. B. Gene distribution of each HCR on the phylogenetic tree and chromosome location. 
We found 8 phylogenetic clusterings for all genes with HCR, which were named and enumerated. Two of these clusters were re-named as CA and CC clusterings. CA corresponds to the ancestral cluster with more than 5 genes, and $\mathrm{CC}$ corresponds to the conserved cluster with a large number of genes ( 49 genes).

All phylogenetic clustering was associated with chromosomal clustering, as shown in Figure 3A, to find a relationship between them. We used this approach to detect relationships between HCR I and HCR II and their possible roles in the function of the gene and their chromosomal distribution (Figure 3B). We found no relationship between the phylogenetic tree topology and most genes with HCR I and HCR II, even with similarity $>70 \%$ in most conserved motifs. This finding suggests that an HCR is not a common feature for a specific gene with the same function and chromosome location. However, the presence of these regions in $25 \%$ of the functional genes is a descriptor parameter to distinguish some members of the OR gene family. It is important to note that of the 62 pseudogenes with an HCR I, 45 are in the CC phylogenetic cluster. However, this clustering is due to the high homology of the HCR rather than the coding sequence of the pseudogenes. Therefore, this cluster was analyzed based on the relationships between the HCR and their chromosome location, not by its phylogenetics arrangement.

\section{DISCUSSION}

Two important points are revealed by this study: i) the presence of an HCR in functional genes and pseudogenes, and ii) the relationship between the function of the genes and their location on the chromosomes and the HCR.

\section{Highly conversed regions}

To analyze these regions, it is important to consider the structural characteristics of each HCR. Position and length of each HCR are common characteristics both in functional genes and pseudogenes. They display high similarity among themselves, suggesting a similar role for these regions and probably a similar set of specific transcriptional binding factors. These regions (HCRs) in conjunction with other molecular mechanisms could determine the gene expression of the hOR family. For example, a previous microarray study revealed that the expression level of the human olfactory genes and of pseudogenes, even in different tissues (Zhang et al., 2007), can be associated with common mechanisms of regulation or common expression patterns. According to this study, we suggest that the presence of HCR in pseudogenes accounts for their expression in the cell, perhaps at the same levels as the functional genes. If one pseudogene is normally expressed, then the HCR and transcription factors would not be associated with the features by which a pseudogene is defined; for instance, having a coding region less than 250 residues long. This hypothesis has been validated with the chromosomal and phylogenetic analyses explained in the next paragraph.

\section{Highly conserved regions, function and genomic distribution of the genes}

Gene distribution in chromosome-specific locations is a very important concept in understanding the molecular process necessary in the development of a function. If a particular 
set of genes has the same position and the same function, it is possible that all characteristics associated with its expression and regulation will be accomplished. Interestingly, the olfactory gene family is characterized by the regular organization of their structures, such as with axons nestled in the epithelium according to the type of receptor that is expressed (Wang et al., 1998; Feinstein and Mombaerts, 2004; Barnea et al., 2004) and to similar genes in the same location on the chromosomes (Zozulya et al., 2001; Glusman et al., 2001; Niimura and Nei, 2003). However, our study shows that HCR I and HCR II are present in different hOR genes and pseudogenes, and they do not have the same functions or locations on the chromosomes. This fact was shown in mice (Hoppe et al., 2006), where common regulatory regions located upstream from the TSS are associated with the topology of the genes in the olfactory epithelium. Therefore, our phylogenetic tree shows that the gene olfactory regulation mechanisms do not depend on the functionality of the gene; perhaps they are associated with the olfactory epithelium, as reported by Hoppe et al. (2006).

\section{CONCLUSIONS}

We found two highly conserved regions upstream from the transcription start sites for $19 \%$ of the human olfactory receptor genes. These regions have a possible enhancer function, they are not associated directly with the function of the gene, and are present in functional genes and pseudogenes. These qualities are important contributions for clarifying the regulation of the hOR genes and can explain the expression of the pseudogenes found recently.

\section{ACKNOWLEDGMENTS}

We gratefully acknowledge the Instituto Colombiano para el Desarrollo de la Ciencia y la Tecnología, Francisco José de Caldas - COLCIENCIAS, from the Republic of Colombia for supporting this project. We also thank Luis Garreta and Ember Martinez for helping us in the data mining, and all members in the BIMAC research group. Finally, we thank Dr. Albert Leyva for his assistance in the preparation of the manuscript.

\section{REFERENCES}

Altschul SF, Madden TL, Schaffer AA, Zhang J, et al. (1997). Gapped BLAST and PSI-BLAST: a new generation of protein database search programs. Nucleic Acids Res. 25: 3389-3402.

Barnea G, O’Donnell S, Mancia F, Sun X, et al. (2004). Odorant receptors on axon termini in the brain. Science 304: 1468.

Buck L and Axel R (1991). A novel multigene family may encode odorant receptors: a molecular basis for odor recognition. Cell 65: 175-187.

Chess A, Simon I, Cedar H and Axel R (1994). Allelic inactivation regulates olfactory receptor gene expression. Cell 78: 823-834.

Crooks GE, Hon G, Chandonia JM and Brenner SE (2004). WebLogo: a sequence logo generator. Genome Res. 14: 1188-1190.

Feinstein P and Mombaerts P (2004). A contextual model for axonal sorting into glomeruli in the mouse olfactory system. Cell 117: 817-831.

Glusman G, Yanai I, Rubin I and Lancet D (2001). The complete human olfactory subgenome. Genome Res. 11: 685-702.

Hinrichs AS, Karolchik D, Baertsch R, Barber GP, et al. (2006). The UCSC Genome Browser Database: update 2006. Nucleic Acids Res. 34: D590-D598.

Hoppe R, Weimer M, Beck A, Breer H, et al. (2000). Sequence analyses of the olfactory receptor gene cluster mOR37 on 
mouse chromosome 4. Genomics 66: 284-295.

Hoppe R, Frank H, Breer H and Strotmann J (2003). The clustered olfactory receptor gene family 262: genomic organization, promoter elements, and interacting transcription factors. Genome Res. 13: 2674-2685.

Hoppe R, Breer H and Strotmann J (2006). Promoter motifs of olfactory receptor genes expressed in distinct topographic patterns. Genomics 87: 711-723.

Kolchanov NA, Ignatieva EV, Ananko EA, Podkolodnaya OA, et al. (2002). Transcription Regulatory Regions Database (TRRD): its status in 2002. Nucleic Acids Res. 30: 312-317.

Lane RP, Cutforth T, Young J, Athanasiou M, et al. (2001). Genomic analysis of orthologous mouse and human olfactory receptor loci. Proc. Natl. Acad. Sci. U. S. A. 98: 7390-7395.

Malnic B, Hirono J, Sato T and Buck LB (1999). Combinatorial receptor codes for odors. Cell 96: 713-723.

Malnic B, Godfrey PA and Buck LB (2004). The human olfactory receptor gene family. Proc. Natl. Acad. Sci. U. S. A. 101: 2584-2589.

Michaloski JS, Galante PA and Malnic B (2006). Identification of potential regulatory motifs in odorant receptor genes by analysis of promoter sequences. Genome Res. 16: 1091-1098.

Niimura Y and Nei M (2003). Evolution of olfactory receptor genes in the human genome. Proc. Natl. Acad. Sci. U. S. A. 100: 12235-12240.

Notredame C, Higgins DG and Heringa J (2000). T-Coffee: A novel method for fast and accurate multiple sequence alignment. J. Mol. Biol. 302: 205-217.

Olender T, Feldmesser E, Atarot T, Eisenstein M, et al. (2004). The olfactory receptor universe - from whole genome analysis to structure and evolution. Genet. Mol. Res. 3: 545-553.

Pavesi G, Mereghetti P, Mauri G and Pesole G (2004). Weeder Web: discovery of transcription factor binding sites in a set of sequences from co-regulated genes. Nucleic Acids Res. 32: W199-W203.

Qasba P and Reed RR (1998). Tissue and zonal-specific expression of an olfactory receptor transgene. J. Neurosci. 18: 227-236.

Sosinsky A, Glusman G and Lancet D (2000). The genomic structure of human olfactory receptor genes. Genomics 70: 49-61.

Tamura K, Dudley J, Nei M and Kumar S (2007). MEGA4: Molecular Evolutionary Genetics Analysis (MEGA) software version 4.0. Mol. Biol. Evol. 24: 1596-1599.

Taylor TD, Noguchi H, Totoki Y, Toyoda A, et al. (2006). Human chromosome 11 DNA sequence and analysis including novel gene identification. Nature 440: 497-500.

Thompson JD, Higgins DG and Gibson TJ (1994). CLUSTAL W: improving the sensitivity of progressive multiple sequence alignment through sequence weighting, position-specific gap penalties and weight matrix choice. Nucleic Acids Res. 22: 4673-4680.

Vassalli A, Rothman A, Feinstein P, Zapotocky M, et al. (2002). Minigenes impart odorant receptor-specific axon guidance in the olfactory bulb. Neuron 35: 681-696.

Wang F, Nemes A, Mendelsohn M and Axel R (1998). Odorant receptors govern the formation of a precise topographic map. Cell 93: 47-60.

Zhang X, De la Cruz O, Pinto JM, Nicolae D, et al. (2007). Characterizing the expression of the human olfactory receptor gene family using a novel DNA microarray. Genome Biol. 8: R86.

Zozulya S, Echeverri F and Nguyen T (2001). The human olfactory receptor repertoire. Genome Biol. 2: research0018.1-0018.12. 